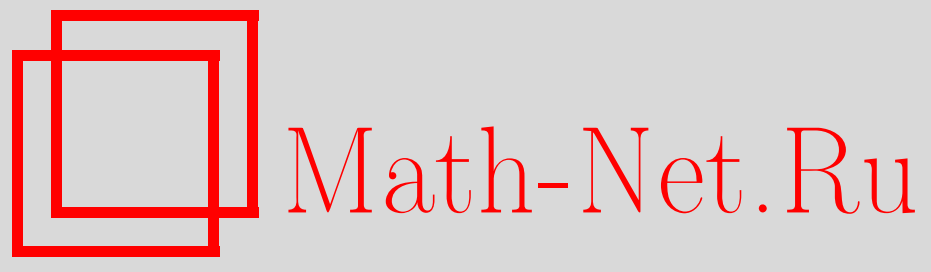

А. В. Цыганов, K задаче Чаплыгина о качении шара, Матем. заметки, 2013, том 94, выпуск 4, 637-640

DOI: https://doi.org/10.4213/mzm9368

Использование Общероссийского математического портала Math-Net.Ru подразумевает, что вы прочитали и согласны с пользовательским соглашением http: //www . mathnet.ru/rus/agreement

Параметры загрузки:

IP: 18.234 .197 .8

26 апреля 2023 г., 16:06:39

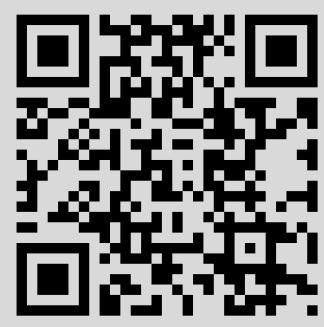




\section{К задаче Чаплыгина о качении шара}

\section{А. В. Цыганов}

Введение. Шаром Чаплыгина называют динамически несимметричное уравновешенное твердое тело со сферической поверхностью, которое катится без проскальзывания по шероховатой плоскости [1]. В подвижной системе координат, связанной с главными осями, угловой момент шара относительно точки касания имеет вид

$$
M=(\mathbf{I}+d \mathbf{E}) \omega-d(x, \omega) x, \quad d=m a^{2} .
$$

Здесь $\omega$ - угловая скорость, $m-$ масса, $\mathbf{I}=\operatorname{diag}\left(I_{1}, I_{2}, I_{3}\right)-$ тензор инерции и $a-$ радиус шара, $x=\left(x_{1}, x_{2}, x_{3}\right)$ - единичный вектор нормали к поверхности шара в точке контакта, a $\mathbf{E}$ - единичная матрица. Скобки $(x, y)$ обозначают обычное скалярное произведение, а $x \times y$-векторное произведение трехмерных векторов. Уравнения движения

$$
\dot{M}=M \times \omega, \quad \dot{x}=x \times \omega .
$$

обладают интегралами движения

$$
H_{1}=(M, \omega), \quad H_{2}=(M, M), \quad H_{3}=(x, x), \quad H_{4}=(x, M)
$$

и инвариантной формой объема

$$
\mu=g^{-1} d x \mathrm{~d} M, \quad g=\sqrt{1-d(x, \mathbf{A} x)},
$$

где $\mathbf{A}=(\mathbf{I}+d \mathbf{E})^{-1}=\operatorname{diag}\left(a_{1}, a_{2}, a_{3}\right)$. Согласно [2] интегралы движения (3) находятся в инволюции относительно скобок Пуассона

$$
\left\{M_{i}, M_{j}\right\}_{g}=\varepsilon_{i j k}\left(g M_{k}-\frac{d(M, \mathbf{A} x)}{g} x_{k}\right), \quad\left\{M_{i}, x_{j}\right\}_{g}=\varepsilon_{i j k} g x_{k}, \quad\left\{x_{i}, x_{j}\right\}_{g}=0,
$$

где $\varepsilon_{i j k}$ - полностью антисимметричный тензор. Уравнения движения (2) после замены времени

$$
d t \rightarrow g d t
$$

становятся гамильтоновыми относительно скобки Пуассона (5).

Приведение скобки Пуассона к канонической. В работе [3] доказано, что скобки Пуассона (5) являются деформацией канонических скобок Ли-Пуассона на алгебре $e^{*}(3)$ в когомологии Пуассона-Лихнеровича. В силу этого должна существовать замена переменных, которая приводит скобки Пуассона (5) для шара Чаплыгина к каноническим скобкам. Нашей основной целью является построение такого преобразования.

Напомним, что канонические скобки Ли-Пуассона для коалгебры $e^{*}(3)$ группы движений трехмерного евклидова пространства имеют вид

$$
\left\{L_{i}, L_{j}\right\}=\varepsilon_{i j k} L_{k}, \quad\left\{L_{i}, x_{j}\right\}=\varepsilon_{i j k} x_{k}, \quad\left\{x_{i}, x_{j}\right\}=0,
$$

и обладают функциями Казимира $(x, x)$ и $(x, L)$.

Если $(x, M)=0$, то скобки Пуассона (5) приводятся к каноническим (7) преобразованием $L_{i}=g^{-1}(x) M_{i}$, см. [2]. На симплектических листах общего положения преобразование выглядит следующим образом:

$$
L_{i}=g^{-1}(x)\left(M_{i}-b h_{i}(x)\right), \quad b=(x, M),
$$

DOI: $10.4213 / \operatorname{mzm} 9368$ 
где функции $h_{i}(x)$ удовлетворяют одному алгебраическому уравнению

$$
c g(x)=1+\sum_{i=1}^{3} x_{i} h_{i}(x) \quad \Longleftrightarrow \quad(x, L)=c(x, M), \quad c \in \mathbb{R},
$$

и одному дифференциальному уравнению

$$
\sum_{i=1}^{3}\left[h_{i}^{2}-\partial_{i} h_{i}+h_{i} \sum_{j \neq i}\left(x_{j} \partial_{i} h_{j}-x_{i} \partial_{j} h_{j}\right)\right]=0, \quad \partial_{k}=\frac{\partial}{\partial x_{k}} .
$$

Так как переменные $x_{k}$ зависимы, то для решения этих уравнений мы воспользуемся стандартным представлением коалгебры $e^{*}(3)$ через углы Эйлера и сопряженные им импульсы

$$
\begin{array}{ll}
x_{1}=\sin \phi \sin \theta, & M_{1}=\left(\frac{\sin \phi}{\sin \theta}\left(\cos \theta p_{\phi}+b\right)-\cos \phi p_{\theta}\right), \\
x_{2}=\cos \phi \sin \theta, & M_{2}=\left(\frac{\cos \phi}{\sin \theta}\left(\cos \theta p_{\phi}+b\right)+\sin \phi p_{\theta}\right), \\
x_{3}=\cos \theta, & M_{3}=-p_{\phi},
\end{array}
$$

и аналогично для переменных $L$. Здесь и всюду далее мы предполагаем, что

$$
x_{1}^{2}+x_{2}^{2}+x_{3}^{2}=1,
$$

а все остальные симплектические листы $e^{*}(3)$ могут быть получены из данного листа преобразованием $x \rightarrow r x$.

Входящие в определение переменных $L_{i}$ импульсы $p_{\phi}$ и $p_{\theta}$ удовлетворяют каноническим скобкам Пуассона

$$
\left\{\phi, p_{\phi}\right\}=\left\{\theta, p_{\theta}\right\}=1, \quad\{\phi, \theta\}=\left\{\phi, p_{\theta}\right\}=\left\{\theta, p_{\phi}\right\}=\left\{p_{\phi}, p_{\theta}\right\}=0,
$$

порождающими скобки (7). С другой стороны, входящие в определение переменных $M_{i}$ импульсы $p_{\phi}$ и $p_{\theta}$ удовлетворяют скобкам Пуассона вида

$$
\begin{aligned}
& \left\{\phi, p_{\phi}\right\}_{g}=\left\{\theta, p_{\theta}\right\}_{g}=g, \quad\{\phi, \theta\}_{g}=\left\{\phi, p_{\theta}\right\}_{g}=\left\{\theta, p_{\phi}\right\}_{g}=0, \\
& \left\{p_{\phi}, p_{\theta}\right\}=\frac{\partial g}{\partial \theta} p_{\phi}-\frac{\partial g}{\partial \phi} p_{\theta}-b d g \cos \theta\left(a_{1} \sin ^{2} \phi+a_{2} \cos ^{2} \phi\right) .
\end{aligned}
$$

Линейное по моментам преобразование

$$
p_{\phi} \rightarrow g^{-1} p_{\phi}+b h_{1}(\phi, \theta), \quad p_{\theta} \rightarrow g^{-1} p_{\theta}+b h_{2}(\phi, \theta),
$$

переводит скобки Пуассона $\{\cdot, \cdot\}_{g}(10)$ в канонические скобки Пуассона $\{\cdot, \cdot\}(9)$, если справедливо уравнение

$$
\left(\frac{\partial h_{1}}{\partial \theta}-\frac{\partial h_{2}}{\partial \phi}\right)-b d g^{-1} \cos \theta\left(a_{1} \sin ^{2} \phi+a_{2} \cos ^{2} \phi\right)=0 .
$$

Для функции $g=\sqrt{1-d\left(a_{1} x_{1}^{2}+a_{2} x_{2}^{2}+a_{3} x_{3}^{2}\right)}$ (см. (4)), отвечающей шару Чаплыгина, уравнение (11) имеет частное решение

$$
h_{2}=0, \quad h_{1}=g^{-1} \cos \theta\left(1-\frac{1}{1-d\left(a_{1} \sin ^{2} \phi+a_{2} \cos ^{2} \phi\right)}\right) .
$$

Заметим, что если $h_{1}=0$ или $h_{1}=h_{2}$, то решения уравнения (11) будут эллиптическими функциями от углов Эйлера $\phi, \theta$. 
Теорема 1. Отвечающее решению (12) пуассоново отображение (8)

$$
\begin{aligned}
& L_{1}=g^{-1}\left(M_{1}+\frac{b d\left(a_{1} x_{1}^{2}+a_{2} x_{2}^{2}\right) x_{1} x_{3}^{2}}{\left(d\left(a_{1} x_{1}^{2}+a_{2} x_{2}^{2}\right)-x_{1}^{2}-x_{2}^{2}\right)\left(x_{1}^{2}+x_{2}^{2}\right)}-\frac{b x_{1}}{x_{1}^{2}+x_{2}^{2}}\right)+\frac{b c x_{1}}{x_{1}^{2}+x_{2}^{2}}, \\
& L_{2}=g^{-1}\left(M_{2}+\frac{b d\left(a_{1} x_{1}^{2}+a_{2} x_{2}^{2}\right) x_{2} x_{3}^{2}}{\left(d\left(a_{1} x_{1}^{2}+a_{2} x_{2}^{2}\right)-x_{1}^{2}-x_{2}^{2}\right)\left(x_{1}^{2}+x_{2}^{2}\right)}-\frac{b x_{2}}{x_{1}^{2}+x_{2}^{2}}\right)+\frac{b c x_{2}}{x_{1}^{2}+x_{2}^{2}}, \\
& L_{3}=g^{-1}\left(M_{3}-\frac{b d\left(a_{1} x_{1}^{2}+a_{2} x_{2}^{2}\right) x_{3}}{\left(d\left(a_{1} x_{1}^{2}+a_{2} x_{2}^{2}\right)-x_{1}^{2}-x_{2}^{2}\right)\left(x_{1}^{2}+x_{2}^{2}\right)}\right)
\end{aligned}
$$

npu

$$
(x, x)=1, \quad(x, L)=c(x, M), \quad(x, M)=b
$$

приводит скобки Пуассона (5) к каноническим скобкам Ли-Пуассона (7).

Таким образом, заменой времени и координат исходная неголономная система (2) приводится к гамильтоновой интегрируемой системе на коалгебре $e^{*}(3)$ с каноническими скобками Пуассона.

Возможные приложения. Если положить $c=0$ в (13), то мы получим пуассоново отображение симплектических листов общего положения скобки Пуассона $\{\cdot, \cdot\}_{g}(5)$ в сингулярные листы канонической скобки Ли-Пуассона $\{\cdot, \cdot\}(7)$ при

$$
(x, x)=1, \quad(x, L)=0,
$$

которые симплектоморфны кокасательному расслоению $T^{*} S$ сферы единичного радиуса. В этом случае, заменяя в интегралах движения (3) переменные $M$ на $L$ и используя затем сферо-конические координаты на сфере $u_{1,2}$

$$
x_{i}=\sqrt{\frac{\left(u_{1}-e_{i}\right)\left(u_{2}-e_{i}\right)}{\left(e_{j}-e_{i}\right)\left(e_{k}-e_{i}\right)}}, \quad L_{i}=\frac{-2 \varepsilon_{i j k} x_{j} x_{k}\left(e_{j}-e_{k}\right)}{u_{1}-u_{2}}\left(\left(e_{i}-u_{1}\right) p_{1}-\left(e_{i}-u_{2}\right) p_{2}\right),
$$

при $e_{i}=d I_{i}^{-1}$, мы получим

$$
\begin{aligned}
& H_{1}=\mathrm{a} p_{1}^{2}+2 \mathrm{~b} p_{1} p_{2}+\mathrm{c} p_{2}^{2}+\mathrm{d} p_{1}+\mathrm{e} p_{2}+\mathrm{f} \\
& H_{2}=\mathrm{A} p_{1}^{2}+2 \mathrm{~B} p_{1} p_{2}+\mathrm{C} p_{2}^{2}+\mathrm{D} p_{1}+\mathrm{E} p_{2}+\mathrm{F} .
\end{aligned}
$$

Здесь $p_{1,2}$ - моменты сопряженные координатам $u_{1,2}$, а коэффициенты полиномов в (14) являются аналитическими функциями от $u_{1,2}$. Согласно [4] гамильтоновы системы с такими интегралами допускают частичное разделение переменных в уравнении ГамильтонаЯкоби. Переменные разделения $s_{1,2}$ в этом случае являются корнями уравнения

$$
(\mathrm{B}-\mathrm{b} s)^{2}-(\mathrm{A}-\mathrm{a} s)(\mathrm{C}-\mathrm{c} s)=0,
$$

которые в нашем случае равны $s_{1,2}=d\left(1+u_{1,2}\right) u_{1,2}^{-1}$. Как и для случая Клебша [4], эти переменные эволюционируют на примиане алгебраической кривой, которая не является гиперэллиптической, в отличие от разделения переменных у Чаплыгина [1].

Практически для всех известных гамильтоновых интегрируемых систем на коалгебре Ли $e^{*}(3)$ можно построить несколько различных матриц Лакса, используя метод сдвига аргумента или метод классической $r$-матрицы. Мы надеемся, что эти же методы могут быть использованы для построения матрицы Лакса для гамильтоновой системы на $e^{*}(3)$, отвечающей шару Чаплыгина и, тем самым, для самого шара Чаплыгина. Кроме того, известно, что канонические скобки Ли-Пуассона (7) совместны с несколькими другими скобками Пуассона. Применяя к этим скобкам обратное к (13) отображение мы получим набор скобок совместных со скобками Пуассона (5) для шара Чаплыгина. 


\section{СПИСОК ЦИТИРОВАННОЙ ЛИТЕРАТУРЫ}

[1] С. А. Чаплыгин, Собрание сочинений. Т. 1. Теоретическая механика. Математика, Гостехиздат, М.-Л., 1948, 76-101. [2] А. В. Борисов, И. С. Мамаев, Матем. заметки, 70:5 (2001), 793-795. [3] А. V. Tsiganov, J. Geom. Mech., 3:3 (2011), 337-362. [4] В. Г. Марихин, В. В. Соколов, Нелинейная динам., 4:3 (2008), 313-322.

\section{А. В. Цыганов}

Санкт-Петербургский государственный университет

E-mail: andrey.tsiganov@gmail.com
Поступило

13.02 .2012

Исправленный вариант 22.01 .2013 